This paper was published in proceedings 6290 and is made available as an electronic reprint with permission of SPIE. One print or electronic copy may be made for personal use only. Systematic or multiple reproduction, distribution to multiple locations via electronic or other means, duplication of any material in this paper for a fee or for commercial purposes, or modification of the content of the paper are prohibited.

\title{
Beam Shaping for Relay Mirrors
}

\author{
Justin D. Mansell \\ MZA Associates Corporation, 2021 Girard Ste 150, Albuquerque, NM 87106
}

\begin{abstract}
Relay mirrors offer a method of extending the range and efficacy of laser weapons systems by providing a platform that can relay the high power beam from the source to a target at a lower cost than building more laser weapons. Most laser weapons' platforms use a Cassegrain aperture telescope to project the high-power beam to a target. Diffraction causes power in the projected beam to move out beyond the edge of the receiving aperture and into the central obscuration of the receiving Cassegrain telescope. This paper presents a method of increasing the power coupling between a projected beam and a receiving telescope by warping the phase of the projected beam and shows that the spatial frequency and amplitude of the phase aberration is consistent with being integrated with the adaptive optics control loop on the source beam projector.
\end{abstract}

Keywords: Wave-optics, lasers, relay mirrors, optical system modeling, WaveTrain, beam shaping.

\section{INTRODUCTION}

Beam shaping using spatial phase control has been studied and demonstrated for many years. ${ }^{1}$ Applications have included everything from particle trapping to novelty laser pointer beam shaping. Researchers have used a wide variety of devices to achieve beam shaping, including holographic optical elements (HOEs), deformable mirrors, and binary optics. In all of these cases, the wavefront of a beam of light is modified and then allowed to propagate a distance (often to the far-field) to enact the intensity profile change.

In this paper the use of beam shaping for optimizing the power coupling between two telescopes across a distance is explored. The first section describes the problem of relaying light between two telescopes and the applications for such a relay. The next section describes the algorithm used to find a phase profile for beam shaping, the limitations imposed to make the solution enabling, the resulting beam shaping profile, and the results of the wave-optics modeling of the beam shaping. The final sections address the effect of the beam shaping on the beam quality and conclude.

\section{RELAY MIRRORS}

The military has been developing directed energy (DE) weapons for several decades. The cost of a single DE weapon is expected to prohibit mass fabrication. For this reason, researchers developed the concept of a relay mirror extending the range of a single DE weapon at a lower cost. Figure 1 is a schematic of a general relay-mirror engagement. Light from a source is transmitted through the optical train and through the atmosphere to the relay. The source can be a stationary ground-based platform, for example, the Theater High Energy Laser (THEL); an oceanic platform such as a ship or a submarine; or on an aerial platform such as the Airborne Laser (ABL). The optical train will typically contain wavefront control elements, such as steering mirrors and deformable mirrors, and diagnostic sensors, such as wavefront sensors and position sensitive detectors. These beam control systems are used to compensate for aberrations inherent to the source and for aberrations induced by the atmosphere. The final optics in the beam train on the platform are typically a Cassegrain telescope for beam expansion and low-speed focus control. The relay platform contains a Cassegrain telescope for receiving the beam. After the beam has been reduced in size it can be conditioned by another beam control system before being transmitted out to a target.

A team lead by Ball Aerospace demonstrated the relay mirror concept with an orbiting satellite relay mirror about 15 years ago. ${ }^{2}$ It is currently being investigated by the Air Force in a project called the Aerospace Relay Mirror System (ARMS) with the end goal of using a high-altitude airship (HAA) instead of an orbiting satellite. Although this

\footnotetext{
*jimansell@mza.com; phone 1505 245-9970 x122
} 
paper was written to address the DE relay mirror problem, uses of this technique can be found in other applications such as both free-space optical communications and deep-space optical communications.

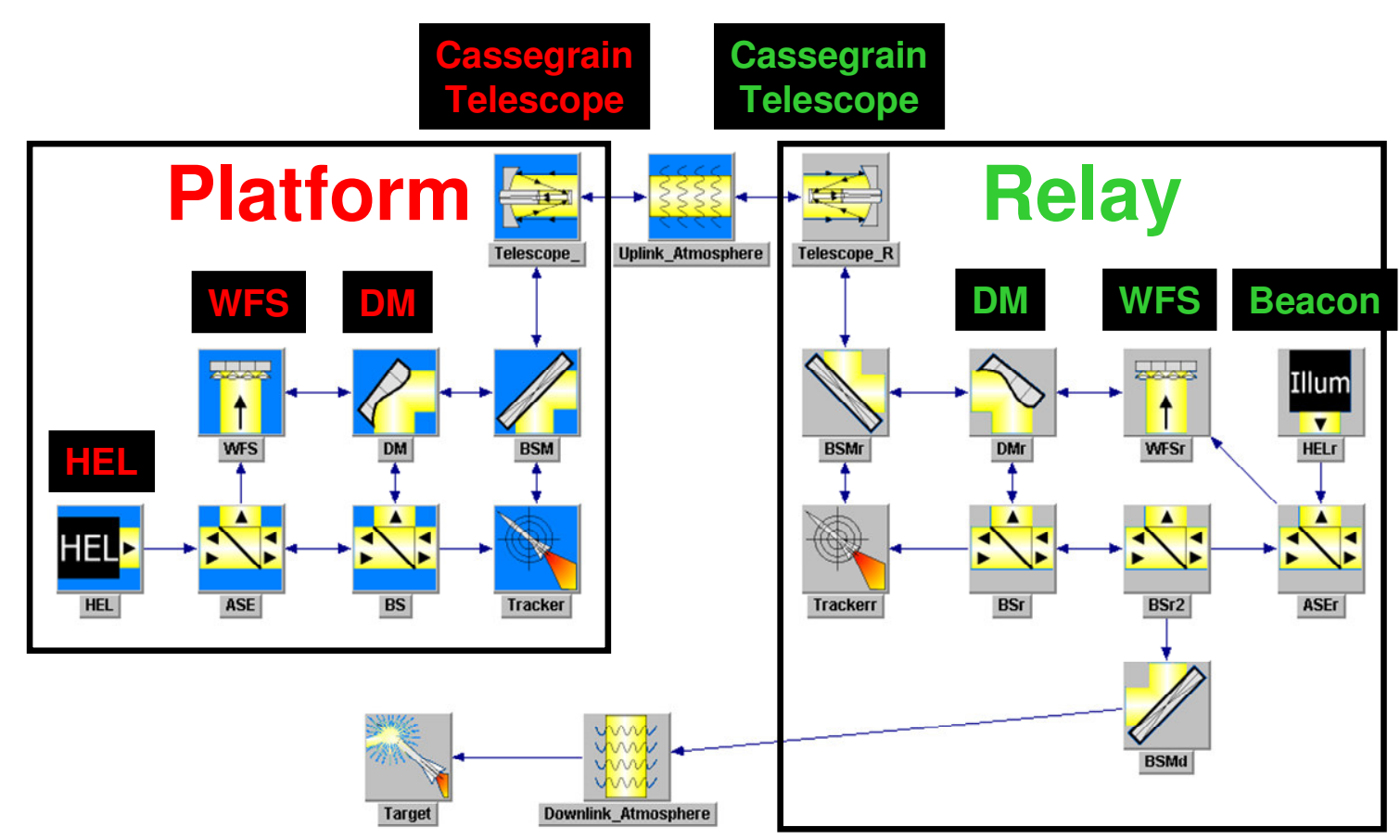

Figure 1 - Schematic of a typical relay mirror engagement.

Unfortunately, in a typical relay mirror engagement aperture diffraction and atmospheric turbulence both reduce the amount of light that is received by the relay telescope. In this first set of simulations, a vacuum propagation was performed between the platform and relay apertures to isolate the effect of aperture diffraction.

In a Cassegrain telescope, the secondary mirror forms a central obscuration (CO) for the light entering or leaving the telescope. As light propagates from a Cassegrain aperture, it tends to diffract into the central obscuration. If a Cassegrain aperture is used on the receiver (relay) side, light diffracted into the central obscuration will be lost. Wave-optics simulations were executed to determine the magnitude of the effect of the central obscuration on the receiver telescope. In each of these experiments, a beam being transmitted from a 1.5-m diameter Cassegrain telescope was propagated to a 1.5-meter diameter telescope on the receiver. In the first set of experiments, a central obscuration was used on both sides. In the second set, it was only used on the transmitter side. The central obscuration was varied in diameter from 0.3 to 0.75 meters. When a central obscuration was used on the receiver telescope, it was the same size as the one on the transmitter telescope. A wavelength of $1.3 \mu \mathrm{m}$ and a telescope diameter of $1.5 \mathrm{~m}$ were used throughout these simulations. The wave-optics propagation parameters were determined for each case using the theory presented by Coy. ${ }^{3}$

Figure 2 shows the results of the simulations with and without a central obscuration on the receiver (relay) telescope for a set of ranges from $50 \mathrm{~km}$ to $400 \mathrm{~km}$ (the range of relay propagation distance throughout this study). For short distances where the effects of diffraction are not strong, the power coupling is quite high for both cases. As the propagation distances get longer, the central obscuration blocks more of the transmitted light. In the longest propagation case of $400 \mathrm{~km}$ when using the largest central obscuration, the receiver telescope with a central obscuration only receives $29 \%$ of the light. Without a central obscuration it receives $59 \%$ of the transmitted light. In this case, $30 \%$ of the light is lost because of the central obscuration of the Cassegrain aperture. Analysis of these results prompted the consideration of beam shaping methods to possibly compensate for some of the diffraction loss on the central obscuration. 


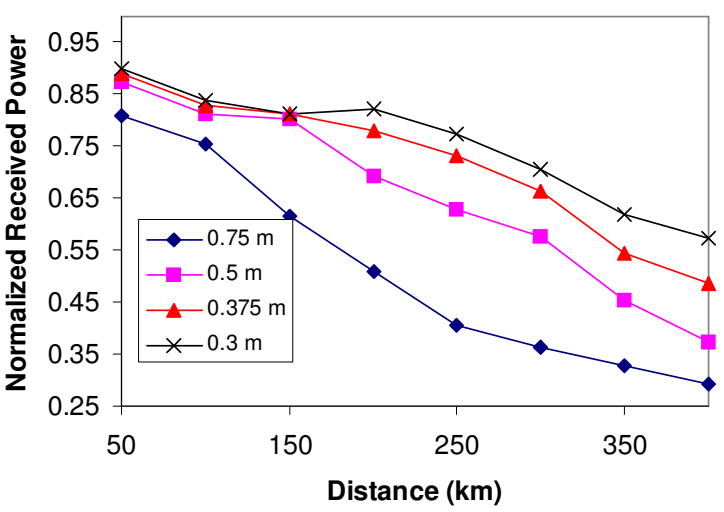

(a)

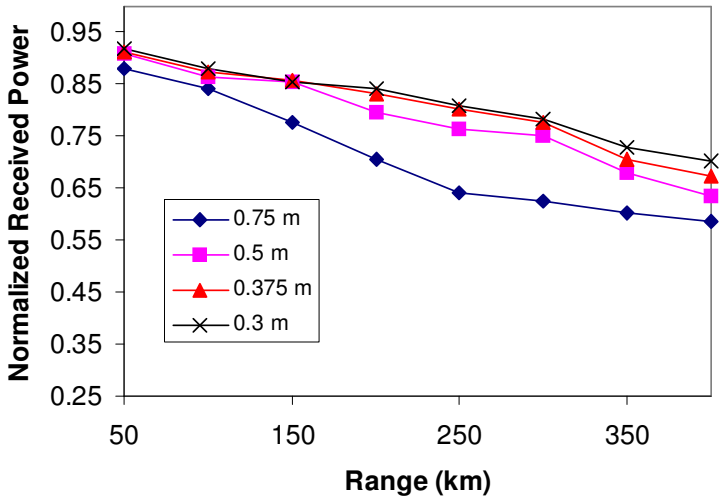

(b)

Figure 2 - Normalized received power for vacuum propagation from a Cassegrain aperture to an aperture with (a) and without (b) the central obscuration on the receive telescope.

\section{BEAM SHAPING}

Although many methods have been demonstrate for beam shaping, some are better for high power laser applications than others. Small transmissive or reflective optics are often not appropriate because the power densities are too high to avoid damage or significant thermally induced distortion of the optic. Furthermore, manufacturing larger beam shaping optics is often very costly.

For this paper, deformable mirrors were considered as a possible beam shaping element. Since most transmitter platforms already have a deformable mirror in place, it may be possible to use them for beam shaping in parallel with atmospheric aberration compensation. However, the deformable mirror must have sufficient range to accomplish both tasks of beam shaping and atmospheric aberration compensation and the beam shaping phase profile must not have spatial frequencies that exceed the capability of the deformable mirror. In the following sections, the spatial frequency of the beam shaping pattern is, first, analyzed using diffraction theory and, next, explored using wave-optics modeling. Then, the amplitude of the beam shaping pattern is analyzed.

\subsection{Spatial Frequency}

One problem with deformable mirrors is that they cannot achieve the high spatial frequency patterns that are possible in diamond turned, etched, or holographic optics. Therefore, analysis was done to determine how much spatial frequency content was necessary to do beam shaping. First, simple diffraction theory was used to determine the maximum possible spatial frequency of the aberration pattern. For a given source projection telescope, the diffraction-limited minimum spot size on target is given by,

$$
D_{\text {target }}=2.44 \frac{f \lambda}{D_{\text {projection }}}
$$

where $\mathrm{D}_{\text {target }}$ is the diameter of the spot on the target, $\mathrm{D}_{\text {projection }}$ is the diameter of the projection aperture, $\mathrm{f}$ is the distance between the source and the target, and $\lambda$ is the wavelength of light. For light with a 1.3- $\mu \mathrm{m}$ wavelength being projected through a $1.5-\mathrm{m}$ diameter aperture at a distance ranging from $50 \mathrm{~km}$ to $400 \mathrm{~km}$, the diffractionlimited spot size ranges from 0.1 to $0.8 \mathrm{~m}$. This corresponds to a telescope diameter to spot diameter ratio of 15 to 2. If it is assumed that each diffraction-limited spot can create a half-cycle of a sinusoidal oscillation, this corresponds to between 7.5 and 1 cycles per telescope aperture. Therefore, this study was limited to looking at polynomials with orders less than 8 . Since the apertures considered were radially symmetric, no angularly dependent phase component was considered. This limitation also ensures that the results will be of sufficiently limited spatial-frequency to allow a deformable mirror to create them. 


\subsection{Radial Polynomial Wave-Optics Power Coupling Study}

Once a maximum polynomial order was determined using diffraction theory, a wave-optics study was done to determine the magnitude of the effect of each successive radial polynomial in increasing the coupling of light from one telescope to the other. For this study, a polynomial phase profile was constructed using the equation,

$$
\phi(r)=\sum_{i=1}^{N} c_{i} \cdot\left|r^{i}\right|
$$

where $\varphi$ is the optical phase profile, $\mathrm{N}$ is the maximum order, $\mathrm{c}_{\mathrm{i}}$ is the coefficient of the radial polynomial, and $\mathrm{r}$ is the radius. The absolute value of the radial power was taken to avoid axial asymmetry. This radial phase polynomial does not represent an orthogonal set spanning the entire space. It was chosen for convenience and to help limit the spatial frequency, permitting better modeling of the limited deformable mirror phase profile.

For this study, a uniform intensity beam of light is transmitted from a Cassegrain aperture to another Cassegrain telescope at the receiver (relay). For the remainder of this paper, the ratio of the size of the Cassegrain telescope aperture to the size of the central obscuration was set to 5 , thereby making the central obscuration $0.3 \mathrm{~m}$. Distances between 50 and $400 \mathrm{~km}$ were studied. For each distance, a study was performed varying the maximum polynomial order between 0 and 6 . (Although the diffraction theory indicated that up to $8^{\text {th }}$ order might be necessary, simulations showed very little change in the coupling capability after $4^{\text {th }}$ order, so the tests were stopped at $6^{\text {th }}$ order.) In each test case, a genetic optimization algorithm called Guided Evolutionary Simulated Annealing (GESA) was used to determine an optimum for the coefficients of the polynomials to maximize the power coupling between the telescopes. ${ }^{4}$ This algorithm will not be guaranteed to find the optimum solution, but is very good at searching through the complex multi-dimensional space of the polynomial coefficients.

Figure 3 illustrates the normalized power coupling while increasing the maximum radial power for various distances. For each of these distances, the increase in normalized power coupling seems to level off. Usually, the increase actually levels off at a radial power of 2, but in some cases ( $250 \mathrm{~km}$ and $300 \mathrm{~km}$ specifically) the power increases by going up to a radial power of 4 . In most of these cases, the increase in power coupling is greater than $5 \%$ and in some cases is more than $12 \%$.

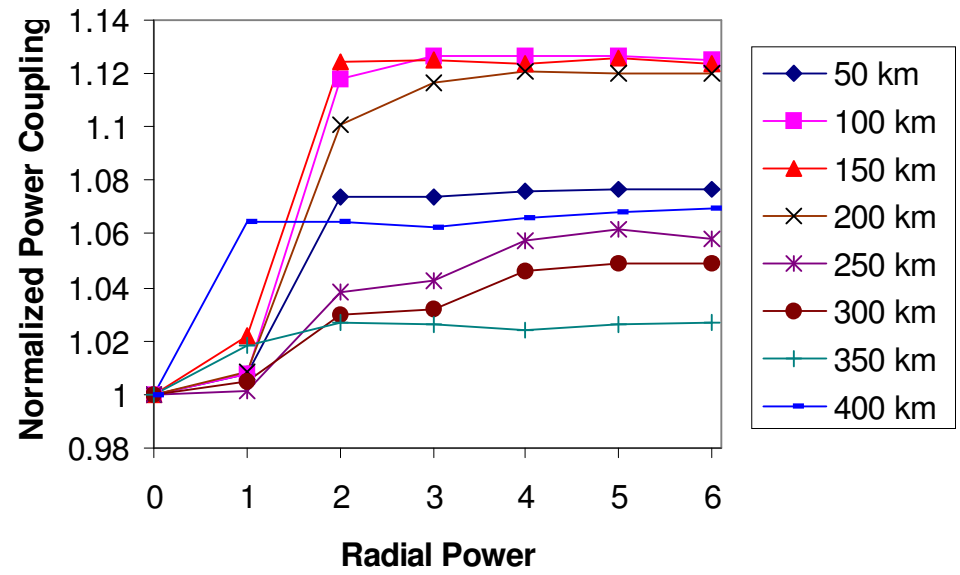

Figure 3 - Effect of added radial power terms on normalized received power between two Cassegrain apertures at varying distances 


\subsection{Optimizing Coefficients of Second and Fourth Order Radial Terms Only}

Since the addition of radial terms beyond $4^{\text {th }}$ order did not significantly increase the power coupling, further study was limited to considering the coefficients of $4^{\text {th }}$ and $2^{\text {nd }}$ order terms alone.

Figure 4 illustrates the power increase factor with respect to range determined by optimizing the coefficients of the $2^{\text {nd }}$ and $4^{\text {th }}$ order radial terms. The results of this study showed that when using just the $2^{\text {nd }}$ and $4^{\text {th }}$ order radial polynomials, the power coupling increased between these apertures by an average of $7.5 \%$ and $12 \%$ in the $100 \mathrm{~km}$ to $200 \mathrm{~km}$ range.

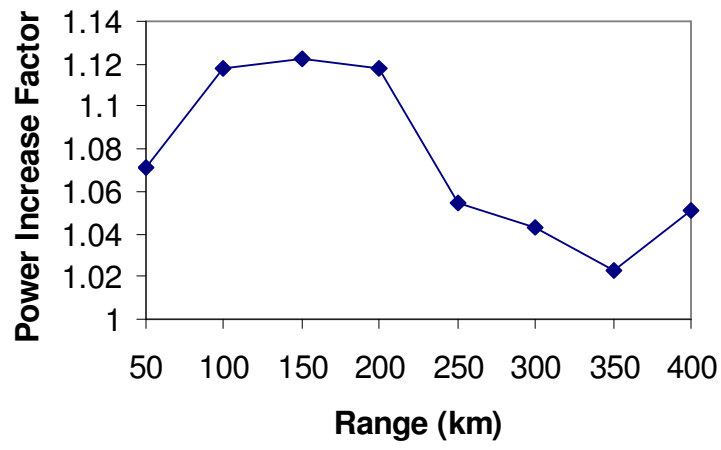

Figure 4 - Increase in power coupling with respect to range for the optimizations of the coefficients of the fourth and second order radial bolvnomials.

Figure 5 shows the optimum coefficients of the $2^{\text {nd }}$ and $4^{\text {th }}$ order terms. In analyzing the results, it was found that the average ratio of the optimized coefficients of the second and fourth order terms was -0.922 with a standard deviation of 0.141 . The mathematical form of the spherical aberration Zernike polynomial is $6 r^{4}-6 r^{2}+1.5$ Since this equation has the same ratio of $2^{\text {nd }}$ and $4^{\text {th }}$ order terms, it was used in subsequent studies as the phase screen shape.

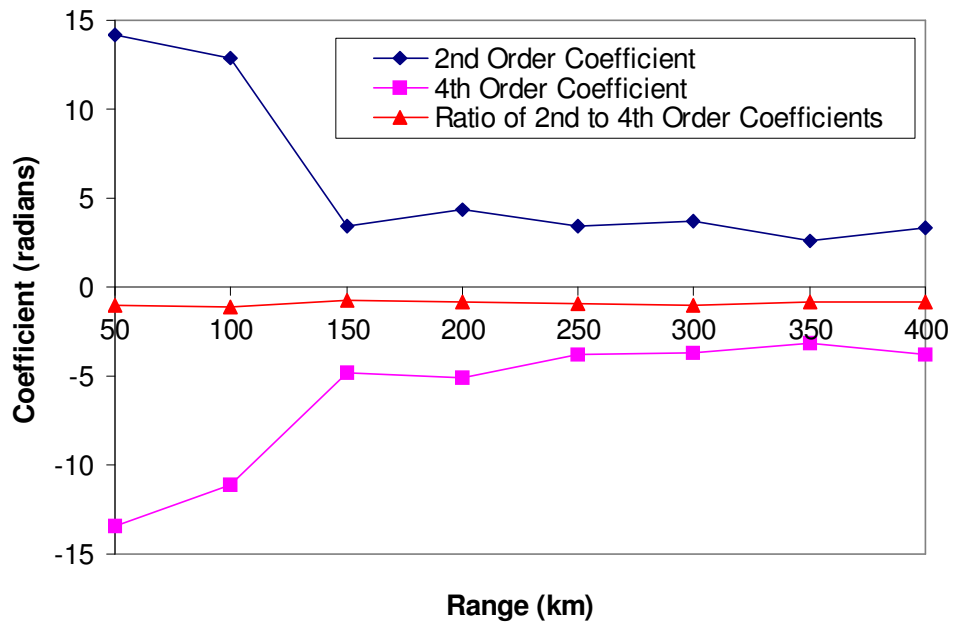

Figure 5 - Results of optimizing the power coupling by adjusting the coefficients of the 2nd and 4th order radial terms. 


\subsection{Beam shaping with only Spherical Aberration in Vacuum}

Since the typical beam shaping profile was on average identical in proportion to the Zernike term for spherical aberration, a model was setup to optimize the coefficient of this term alone. This reduced the number of free parameters and allowed for a more rapid search. In fact, a one-dimensional scan was used to find the optimum coefficient at each length. Figure 7 and Figure 6 show the results of these simulations with and without a central obscuration on the receiver telescope. As the propagation length increased, the amount of spherical aberration necessary to obtain an optimum coupling decreased due to the longer distance over which it had to act. However, even for the shortest range, the amplitude of the spherical aberration for a receiver telescope aperture central obscuration is about 2.5 waves. This corresponds to a deformable mirror throw of 1.25 waves or $1.6 \mu \mathrm{m}$, which is easily achievable for a typical deformable mirror with $7 \mu \mathrm{m}$ (5.4 waves) of throw. This even provides sufficient remaining throw to compensate for atmospheric aberrations in typical engagements. This means that the beam shaping could be integrated with the source adaptive optics system with minor software changes in the control algorithm.

The results also indicate that for shorter distances, the light diffracting into the receiver aperture central obscuration can be affected. But the fact that there is an increase in received power for longer distances seems to indicate that for longer distances the added phase profile is more effective at pushing light from outside the receiver telescope aperture back into the aperture.

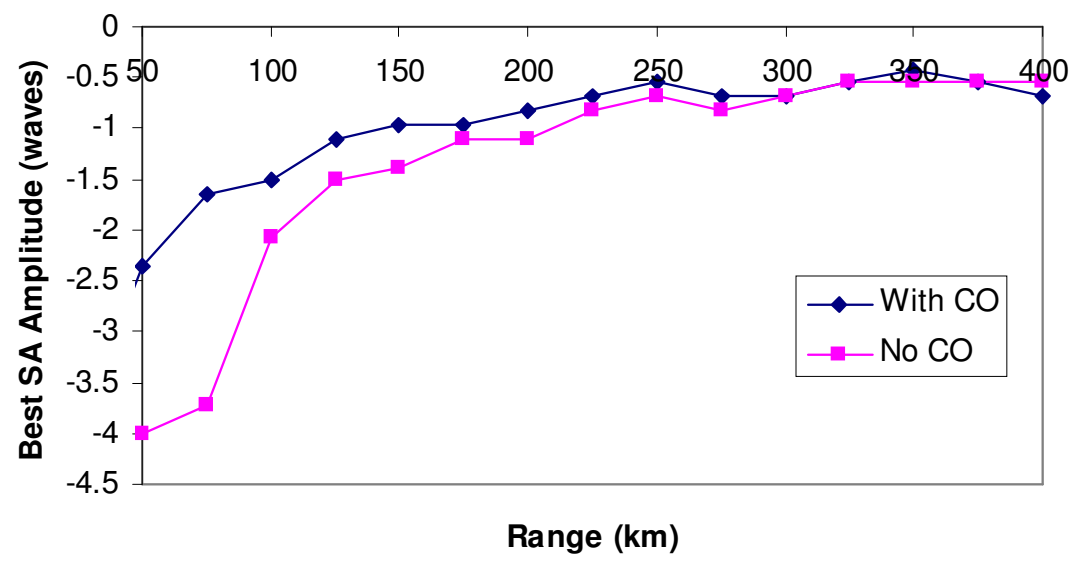

Figure 7 - Optimum spherical aberration coefficient for each range studied.

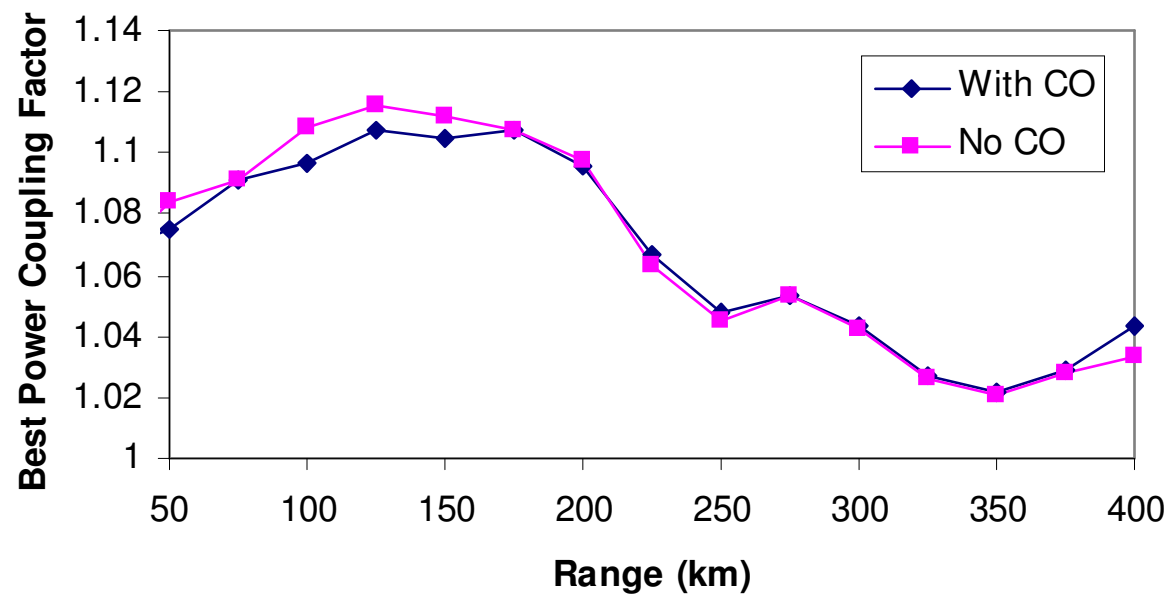

Figure 6 - Optimum power coupling increase factor with respect to range for an optimized spherical aberration phase. 


\section{BEAM SHAPING WITH SPHERICAL ABERRATION THROUGH THE ATMOSPHERE}

The previous simulations assumed that the light was being propagated through a vacuum to the receiver, but in practice this is rarely the case. For DE weapons applications, the light needs to propagate though the atmosphere, which aberrates the beam. The next wave-optics simulation preformed addressed the question of whether the atmospheric induced aberrations would ruin the gains seen in the vacuum propagation cases.

Figure 8 is the WaveTrain simulation setup that runs both the atmospheric and vacuum propagation cases in parallel for a receiver telescope with and without a central obscuration using a spherical aberration Zernike polynomial as the phase screen for power coupling optimization. The first run was done with vacuum propagation only to determine the optimum amount of spherical aberration for each distance. Then, a series of runs were done using 50 random Kolmogorov phase screens with the source on an aerial platform at a $12 \mathrm{~km}$ altitude transmitting to a target at a $21 \mathrm{~km}$ altitude using a Clear- $1 \mathrm{C}_{\mathrm{n}}{ }^{2}$ turbulence profile composed of 5 phase screens evenly distributed along the path. ${ }^{6}$

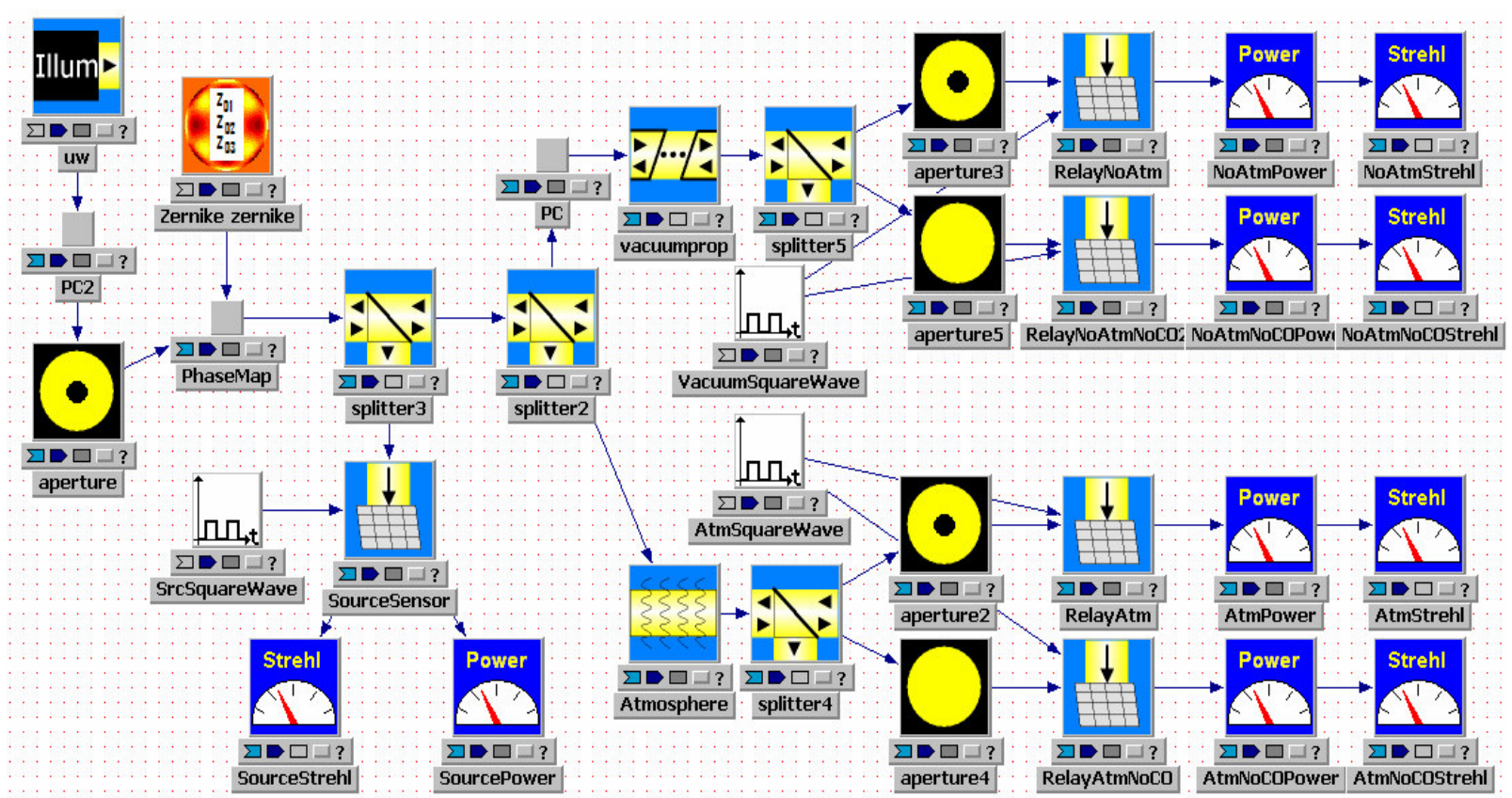

Figure 8 - WaveTrain system setup for modeling relay mirror engagements with and without atmospheric aberrations and with and without a central obscuration in the receiver. 
Figure 9 shows the average power coupling increase using the optimum amount of spherical aberration determined during the vacuum propagation runs with respect to propagation distance. The results are very similar to those in the vacuum propagation study, which indicates that the atmospheric turbulence does not seem to degrade the increase in power coupling gained by adding a beam shaping phase profile to the transmitted beam.

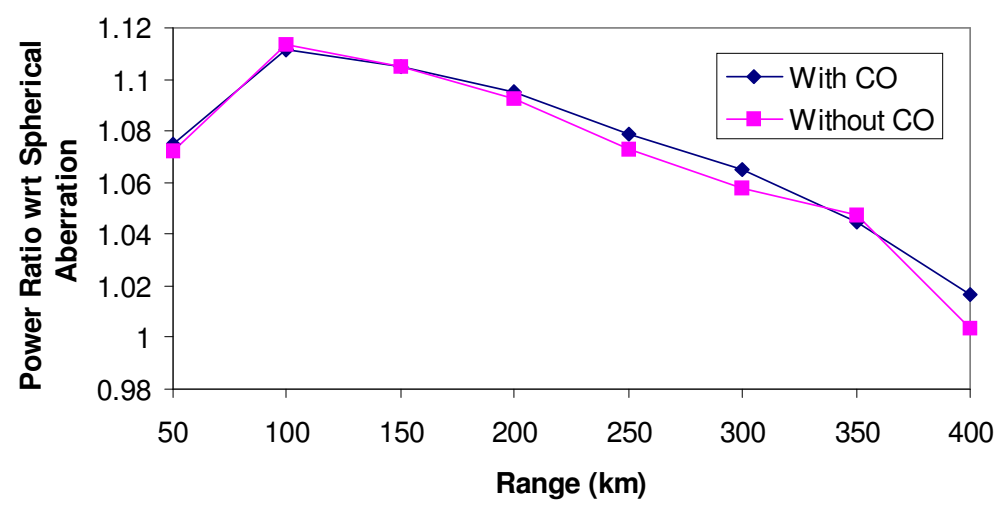

Figure 9 - Normalized power coupling increase with respect to propagation distance through the atmosphere while using the optimum spherical aberration determined with vacuum propagation modeling.

\section{EFFECT OF BEAM SHAPING ON BEAM QUALITY}

Unfortunately, the increase in power coupling does come at the expense of beam quality. Figure 10 shows the effect of the optimum coupling phase screen on the beam's Strehl ratio at a receiver with and without a central obscuration. For these simulations, the Strehl ratio was defined as the ratio of the on-axis intensities of the aberrated beam and an unaberrated beam after taking the Fourier transform of the beam to determine the far-field intensity profile. Since the phase required to achieve higher coupling at longer ranges has small amplitude, the effect on beam quality is less.

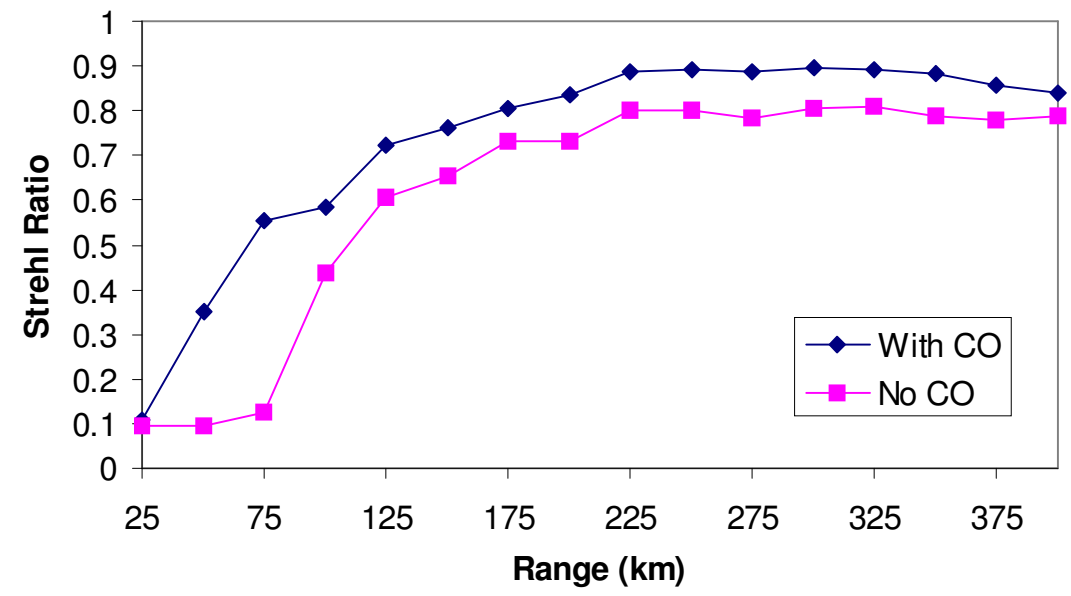

Figure 10 - Strehl ratio at the receiver through vacuum with the optimum spherical aberration with and without the central obscuration in the receiver telescope. 
Figure 11 shows the Strehl ratio of a beam with and without the optimal spherical aberration for enhanced power coupling and with and without the central obscuration on the receiver telescope, measured on the beam, and propagated to the receiver with atmospheric aberrations. This result combines the effects of propagation through the atmosphere, diffraction, and the apertures at both ends. These results were averaged over 50 atmospheric realizations. The results show that the beam quality is reduced even below that caused by the atmospheric effects, but the presence of the central obscuration does not have a significant effect on beam quality.

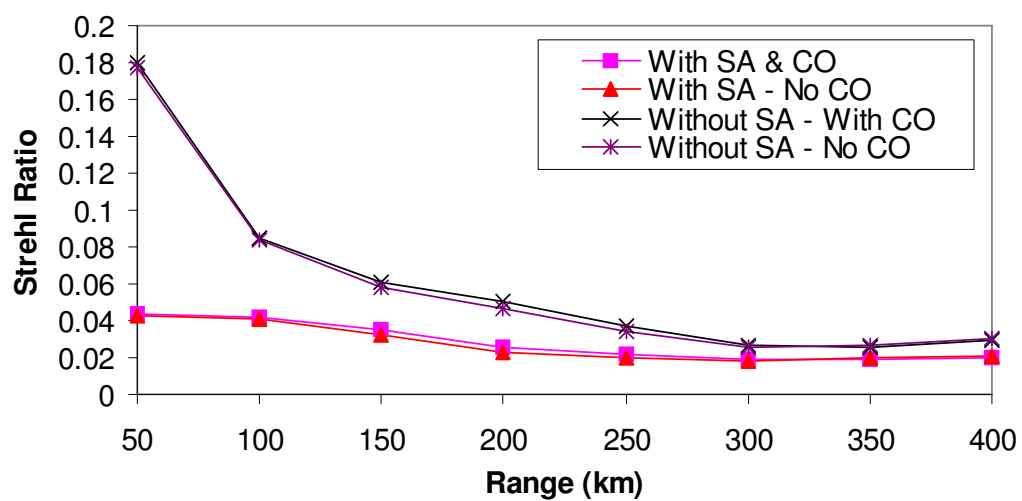

Figure 11 - Average Strehl ratio at the receiver with and without the optimum spherical aberration and with and without the central obscuration in the receiver telescope propagating through the atmosphere.

Despite the reduction in beam quality induced by beam shaping, it is still useful for relay mirror applications. The reduction in beam quality induced by adding the spherical aberration required for enhanced coupling should be easily compensated for by using a low-order beam clean-up adaptive optics system on the relay. The benefits of capturing up to $12 \%$ more power should outweigh the small reduction in beam quality, but more engagement studies need to be done to verify this.

\section{SCALING OF RESULTS}

These results were obtained with a large telescope and a beam propagating over a long distance, but can be applied to a wide range of engagement scenarios by scaling the results by the Fresnel number. The Fresnel number of a propagation is equal to the diameter squared divided by the product of the wavelength and the propagation distance, or $D^{2} / \lambda z$. Figure 12 shows the Fresnel number for the propagation ranges studied in this paper.

\section{CONCLUSIONS}

This paper describes how beam shaping techniques were used to enhance the coupling of a beam of light projected from a Cassegrain telescope at the source into

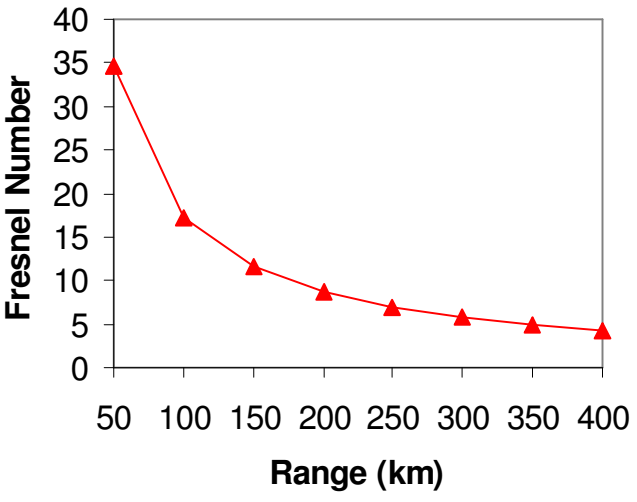

Figure 12 - Fresnel Number of each of the propagation distances in the study.

a Cassegrain telescope at the receiver. The phase profile used in these simulations was initially limited to an $8^{\text {th }}$ order radial polynomial to limit the potential spatial frequency of the beam shaping phase profile to something achievable by conventional continuous phase sheet deformable mirrors, but simulations revealed that limiting the radial polynomial phase screen to $2^{\text {nd }}$ and $4^{\text {th }}$ order radial terms in equal but opposite magnitude parts, which is equivalent in form to the spherical aberration Zernike polynomial, was sufficient to achieve most of the enhancement in the power coupling achieved by the higher-order radial polynomial. Using the spherical aberration phase profile for beam shaping, the power coupling with beam shaping increased by up to $12 \%$ and on average 
7.5\%. These simulations also indicated that the amplitude of the phase profile required was so small that it could probably be put onto the deformable mirror used for atmospheric aberration compensation without having a substantial negative impact on the beam control system's performance in most situations. This beam shaping technique was tested in the presence of atmospheric aberrations and was found to perform, on average, as well as without the aberrations. The only drawback of this technique is the reduction in beam quality that the beam shaping phase profile causes. Since the aberration is relatively low spatial frequency and low amplitude, a beam clean-up adaptive optics control system on the receiver should be easily able to compensate the phase error.

\section{REFERENCES}

${ }^{1}$ F.M. Dickey and S.C. Holswade, Laser Beam Shaping, Marcel Dekker (2000).

2 J.S. Dierks, S.E. Ross, A. Brodsky, P.W. Kervin, and R.W. Holm, "Relay Mirror Experiment overview: a GBL pointing and tracking demonstration", SPIE Vol. 1482, 146-158 (1991).

${ }^{3}$ S. Coy, "Choosing mesh spacings and mesh dimensions for wave optics simulation", SPIE Vol. 5894, paper $5894051-12$.

${ }^{4}$ P. P. C. Yip and Y. Pao. "Growing neural networks using guided evolutionary simulated annealing.", 3rd Annual Conference on Evolutionary Programming (World Scientific, 1994) ,A.V. Sebald and L.J. Fogel, eds., 17-25.

${ }^{5}$ D. Malacara, Optical Shop Testing, Wiley (1992).

${ }^{6}$ Robert R. Beland, "Propagation Through Atmospheric Optical Turbulence" in the Infrared and Electro-Optical Handbook, Vol. 2, Joseph S. Accetta and David L. Schumaker, Editors, ERIM, Ann Arbor, MI and SPIE, Bellingham, WA, 1993. 